\title{
The Color Calibration across Multi-Projector Display
}

\author{
Shu Liu ${ }^{1,2}$, Qiuqi Ruan ${ }^{1,2}$, Xiaoli $\mathrm{Li}^{1,2}$ \\ ${ }^{1}$ Institute of Information Science, Beijing Jiaotong University, Beijing, China; ${ }^{2}$ Beijing Key Laboratory of Advanced Information \\ Science and Network Technology, Beijing, China. \\ Email: Ls87ah@126.com, qqruan@center.njtu.edu.cn,09112087@bjtu.edu.cn
}

Received January $11^{\text {th }}, 2011$; revised March $15^{\text {th }}$, 2011; accepted March $18^{\text {th }}, 2011$.

\begin{abstract}
Large plane displays are often achieved by combining tiled imaging elements, which give the appearance of a single, seamless display. However, this is difficult to realize in practical use, since differences in color will make the tiles distinct even if the geometric calibration is perfect. The paper presents an approach of color calibration using cubic Bspline. According to the theory of cubic B-spline, we construct the color transfer function of each projector. This approach reduces the color and photometric non-uniformities to achieve seamless tiled multi-projector display well. Finally, we propose the difference between blending region and overlapping region which can help to achieve a better effect of alpha blending.
\end{abstract}

Keywords: Multi-Projector Display, Color Calibration, Alpha Blending, Cubic B-Spline

\section{Introduction}

With the continuous development of the projection display technology, the large plane multi-projector displays technique has been widely used in various fields to meet people's increasing demand for high-resolution display. Large plane multi-projector displays are often created by combining tiled imaging elements. In practice, even if the geometric calibration is perfect, it still can not achieve seamless tiled multi-projector display, because of the differences in color.

The problems in achieving color and photometric uniformity of a multi-projector display can be divided into two classes [1].

1) Device Dependent

a) Photometric Variation between Different Projectors;

b) Photometric Variation within a Projector's FOV .

2) Application Dependent

a) Photometric Variation Due to Overlapping Projectors;

b) Photometric Variation Due to Non-planar or NonLambertian Display Surface.

Taking these causes into account, the work of color calibration is carried on in three aspects: calibration within each projector, calibration between the adjacent projectors, and calibration in the overlapping region of projectors. At present, the methods of color calibration mainly fall into two groups. One group is using advanced measuring instruments, such as spectroradiometer [1], colorimeter [2,3], etc. to obtain accurate information for color calibration. The other is extracting information of color intensity from the camera feedback [4,5]. The advantage of the former is that the accurate information obtained by the instruments guarantees the success of color calibration. But the problem is the professional instruments are usually too expensive to be afforded. The latter method greatly reduces the cost, but it brings the drawback of information imprecise at the same time.

This paper presents an approach of color calibration to achieve seamless tiled multi-projector display. One camera is used to get the information of each color channel with different pixel values. Then the projector's color transfer function for each channel is constructed using cubic B-spline.

\section{Background}

\subsection{Color Map}

The color reproduction process of a display system can be described as follow [2]. An RGB triple $(r, g, b)$ in the graphics frame buffer is converted to either an analog voltage or digital bits and sent to the projector. The projector then combines lights of three primary colors proportionally to form the desired color.

The gamut of a display device is the set of all repro- 
ducible colors. The gamut of a color transfer function $F$ can be formally defined as

$$
G(F)=\{F(r, g, b) \mid r, g, b \in[0,255]\}
$$

If it meets the following equation

$$
F(r, g, b)=F(r, 0,0)+F(0, g, 0)+F(0,0, b)
$$

We call the gamut an additive gamut. The three channels RGB are independent of each other.

\subsection{Cubic B-Spline [6]}

The B-spline curve with $u$ and $k$ as its parameter and power respectively can be denoted as follow:

$$
P(u)=\sum_{i=0}^{n} d_{i} N_{i, k}(u)
$$

Where $d_{i}, i=0,1, \cdots, n$ are the control points, and $N_{i, k}(u)$ are the basis function of B-spline. The recursive formula of De Boer-Cox (Cox M.G.) is:

$$
\begin{gathered}
N_{i, 0}(u)= \begin{cases}1, & \text { if } u_{i} \leq u<u_{i}+1 \\
0, \text { else }\end{cases} \\
N_{i, k}(u)=\frac{u-u_{i}}{u_{i+k}-u_{i}} N_{i, k-1}(u)+\frac{u_{i+k+1}-u}{u_{i+k+1}-u_{i+1}} N_{i+1, k-1}(u) \\
k>0, i=0,1, \cdots, n-1
\end{gathered}
$$$$
\text { Set } 0 / 0=0
$$

Given $n$ typical data points $P_{i}(i=0,1,2, \cdots, n-1)$, we can construct the cubic B-spline curve which goes through the $n$ typical data points as follows: if we set the control points as $d_{i}(i=0,1,2, \cdots, n+1)$, the $r^{\text {th }}$ function of the cubic B-spline curve is:

$$
P_{r}(t)=(1 / 6)\left[t^{3}, t^{2}, t, 1\right]\left[\begin{array}{cccc}
-1 & 3 & -3 & 1 \\
3 & -6 & 3 & 0 \\
-3 & 0 & 3 & 0 \\
1 & 4 & 1 & 0
\end{array}\right]\left[\begin{array}{l}
d_{r} \\
d_{r+1} \\
d_{r+2} \\
d_{r+3}
\end{array}\right]
$$

Where $t \in[0,1], r=0,1,2, \cdots, n-2$. Set $P_{r}(0)=P_{r}$, then

$$
(1 / 6) d_{r}=(2 / 3) d_{r+1}+(1 / 6) d_{r+2}=P_{r},
$$

namely $d_{r}+4 d_{r+1}+d_{r+2}=6 P_{r}, r=0,1,2, \cdots, n-1$,. $d_{0}, d_{1}, \cdots, d_{n+1}$ are $n+2$ unknown parameters with only $n$ functions. So we have to add two endpoints premises:

1) For open curve: set $d_{0}=d_{1}, d_{n+1}=d_{n}$;

2) For close curve: set $d_{n+1}=d_{1}, d_{n}=d_{0}$.

\section{Color Transfer Function}

Color matching can be achieved through the color map: $[0,1, \cdots, 255]^{3} \rightarrow[0,1, \cdots, 255]^{3}$, before going through the projector. If the gamut is additive, the color map can be described as $[0,1, \cdots, 255] \rightarrow[0,1, \cdots, 255]$ for each chan- nel. But after the pixels are sent to the display through the projector, the color map is no longer what it was. What we should do is to construct the color transfer function $F$ for the project system.

To be simplified, we assume the gamut additive, thus each channel of the color image is treated separately. In this paper, we only introduce the process of channel $R$. The other two channels, $G$ and $B$, are similar to channel $R$. We can construct the color transfer function of channel $R$ with the following steps.

1) Create 18 images. Set the value of channels $G$ and $B$ to 0 , and sample the value of channel $R$ within $[0,255]$ uniformly spaced of 15 . So we get the sampled values of channel $R \in\{0,15,30, \cdots, 255\}$.

2) Project these 18 images to the display wall in turn, and capture the images on the display wall.

3) Calculate the average pixel value of these 18 photos separately. We regard the average as the response of project for each corresponding pixel value.

4) Calculate the control points using cubic B-spline, with the 18 original pixel values and their response values.

5) According to these control points, construct the color transfer function of the channel $R$ using cubic Bspline. Now we get the color map of channel $R$ of the projector.

\subsection{Color Look-up-Table}

We get the color transfer function of every projector through the above steps. Now we have to linearize the color transfer function for each projector, and match the color of adjacent projectors.

The color transfer function of projector is a non-linear S-curve [7]. What we should do is to correct this nonlinear S-curve into a power function which is approximately linear. The power of function is the Gamma. Generally in order to reflect the true color of the image, we hope the value of gamma is 1 .

We can create the color map through the following steps:

1) Determine the common range of the color output of the projectors, and get the desired transfer function.

$P_{1}, P_{2}$ represent the two adjacent projectors. $F_{1}, F_{2}$ are the color transfer function of $P_{1}, P_{2}$ respectively. In Figure 1, $L_{1}, H_{1}$ are the minimum and maximum response respectively when the pixel is sent by projector $P_{1}$, that is,

$$
\begin{aligned}
& L_{1}=\min \left\{F_{1}(x) \mid 0 \leq x \leq 255, x \in N\right\}, \\
& H_{1}=\max \left\{F_{1}(x) \mid 0 \leq x \leq 255, x \in N\right\}
\end{aligned}
$$

$L_{2}, H_{2}$ are the minimum and maximum response respectively when the pixel is sent by projector $P_{2}$, that is, 


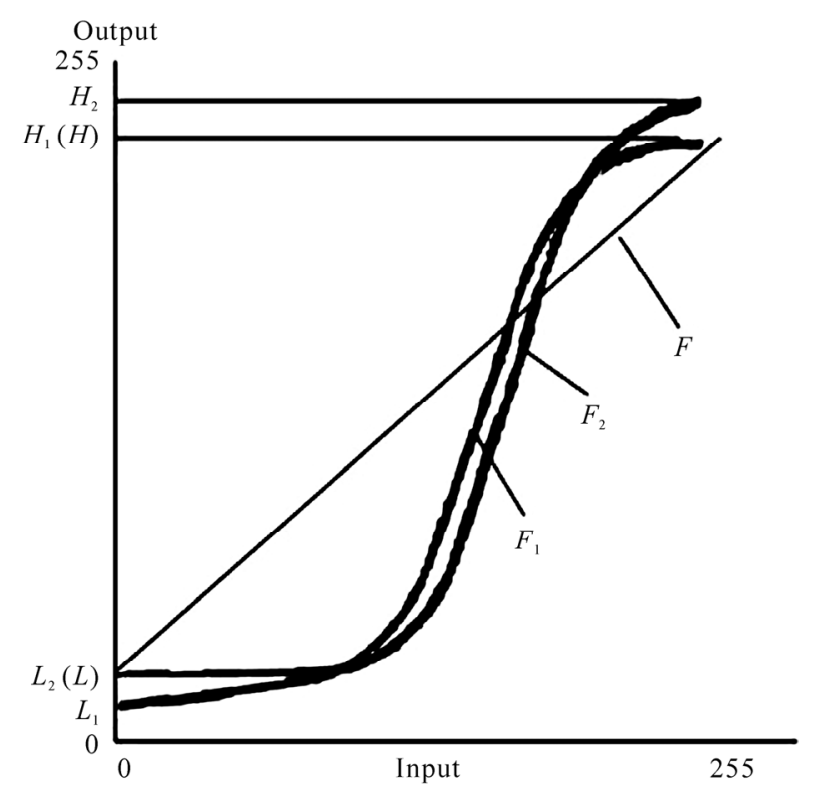

Figure 1. Get the common range of the color output for the projects.

$$
\begin{gathered}
L_{2}=\min \left\{F_{2}(x) \mid 0 \leq x \leq 255, x \in N\right\}, \\
H_{2}=\max \left\{F_{2}(x) \mid 0 \leq x \leq 255, x \in N\right\} \\
L=\max \left\{L_{1}, L_{2}\right\}, \\
H=\min \left\{H_{1}, H_{2}\right\}
\end{gathered}
$$

$F$ is the desired response curve when gamma is 1 ,

$$
F(x)=\frac{H-L}{255} x+L
$$

2) Create the color map, and modify the original pixel value.

In Figure 2, $F_{i}$ is the color transfer function of one projector, and $F$ is the desired response curve. For any pixel $A$ in the image, we expect its corresponding output is $B$. By calculating the inverse function of $F_{i}$, we know the input should be $C$ if $B$ is the output. So we should modify $A$ to be $C$. The formula is

$$
C=F_{i}^{-1}(F(A))
$$

Create color look-up-table (LUT) for each project using the formula. Modify the original pixel with the LUT before the image is sent. In the experiment, we also can do some work about general color calibration, for example the brightness, contrast and gamma correction for each channel. Through the work above, now the color uniformity is achieved.

\subsection{Alpha Blending in Overlapping Regions}

Another important issue need to be addressed is the color

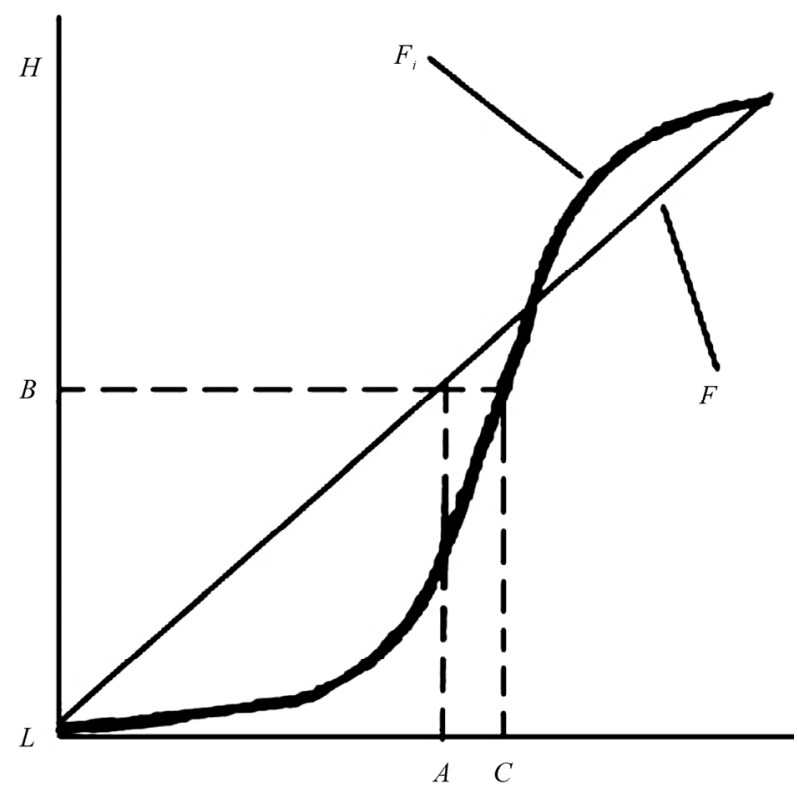

Figure 2. Linearize the original transfer function.

blending of the overlapping regions in the projector display. There will be optical bright band phenomena in the overlapping region if we do nothing with it. The purpose of alpha blending is to eliminate the bright band effect, and achieve seamless tiled multi-projector display.

We add an alpha channel which represents the transparency of the image, because the 24-bit image only contains channels RGB. The value of alpha ranges from 0 to 1 , with 256 levels. When alpha is 1 , the $255^{\text {th }}$ level, it is opaque. When alpha is 0 , the $0^{\text {th }}$, it is transparent. When alpha is between 0 and 1 , it is partially transparent [8]. The next step is to assign different gray values to alpha channel.

In the experiment, we have identified the overlapping region of adjacent projectors and their corresponding positions based on the geometric calibration. For pixels which are not in the overlapping region, their alpha value is 1 . For the others, the alpha value can be calculated as shown in Figure 3 and Figure 4:

Let's normalize the coordinates of the overlapping region into 0 and 1.0 means the beginning of the overlapping region while 1 means the ending. For the image on the right side, the alpha value can be obtained with the following function:

$$
f(x)=\left\{\begin{array}{cc}
0.5(2 x)^{p} & 0 \leq x \leq 0.5 \\
1-0.5(2(1-x))^{p} & 0.5 \leq x \leq 1
\end{array}\right.
$$

For image on the left, the alpha value is $1-f(x)$.

In the overlapping region, the curve can go through the point 0.5 , which means that the pixels in each image have equal contribution to the final pixel value. The precise 


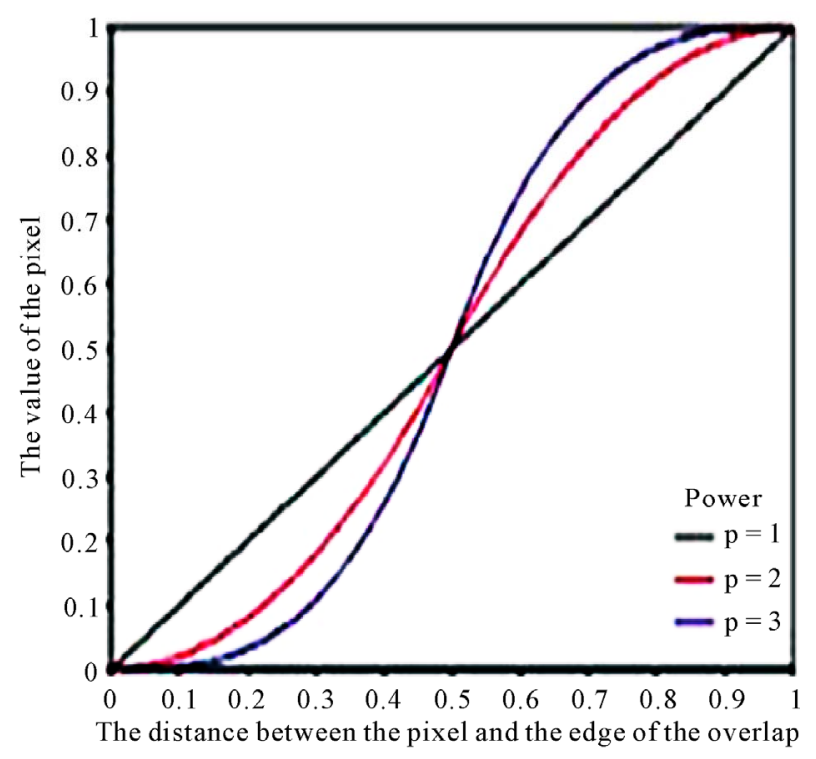

Figure 3. The alpha function $f(x)$ of the right image, when the power takes different values.

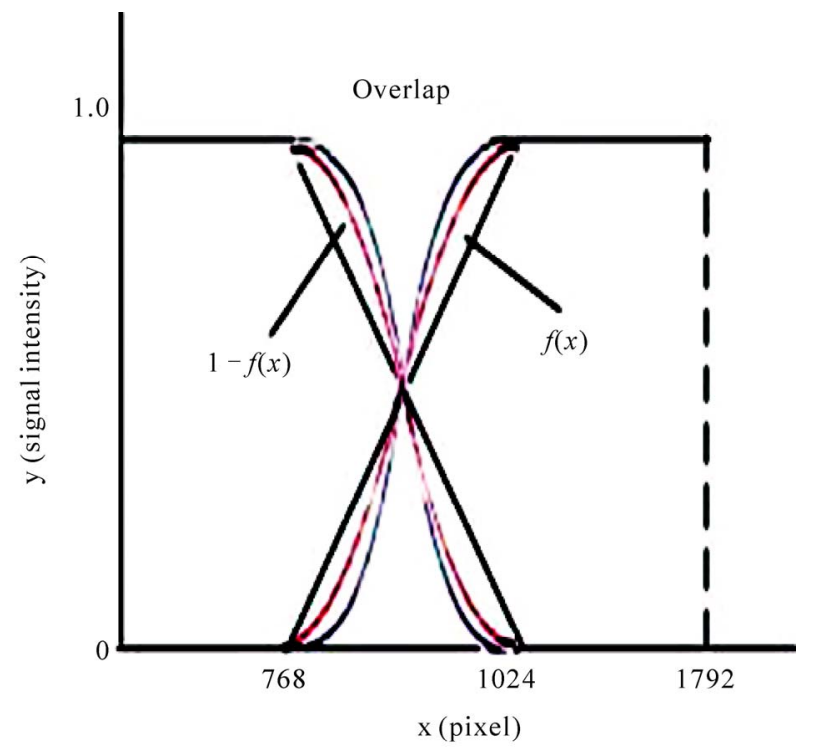

Figure 4. The principle of the edge pixels blend.
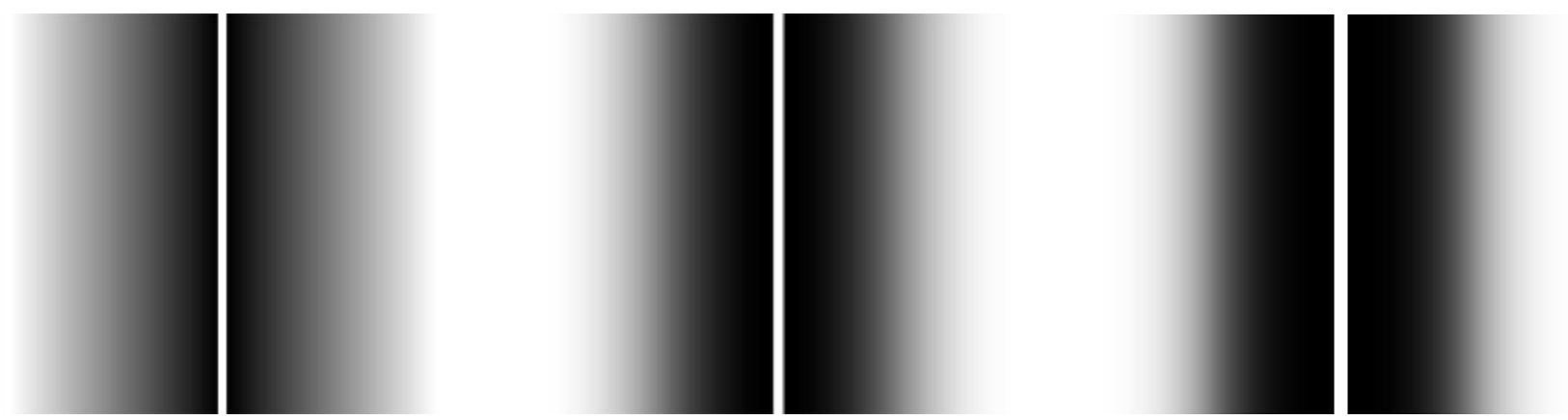

Figure 5. The left is the alpha blending when $p=1$; The middle is the alpha blending when $p=2$; The right is the alpha blending when $p=3$. 

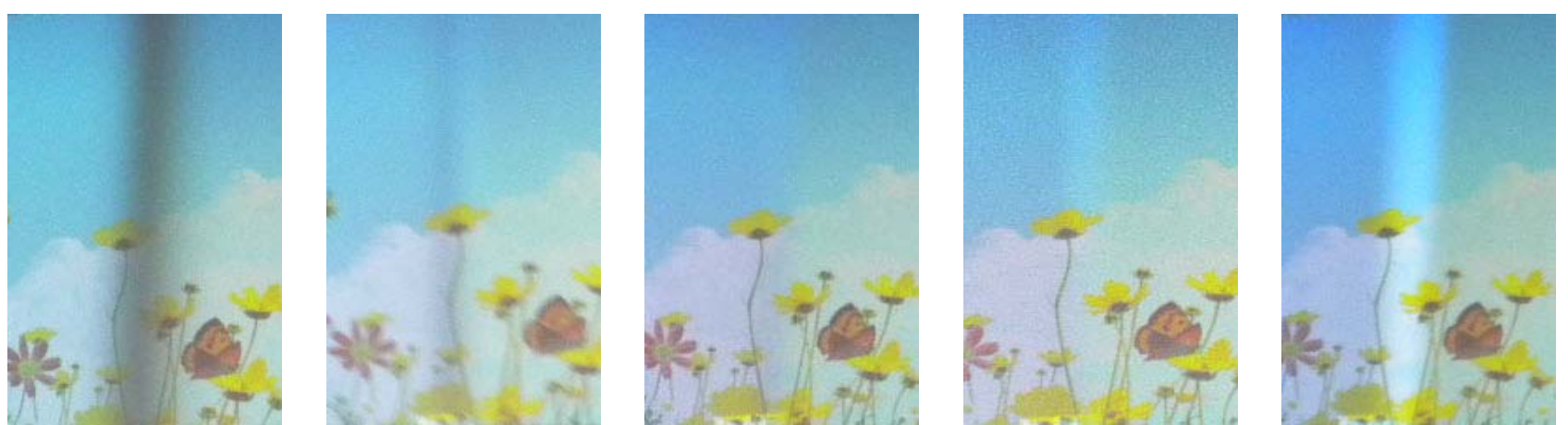

Figure 6. The width of the blending region is 256 pixels, 200 pixels, 180 pixels, 150 pixels and 128 pixels successively.
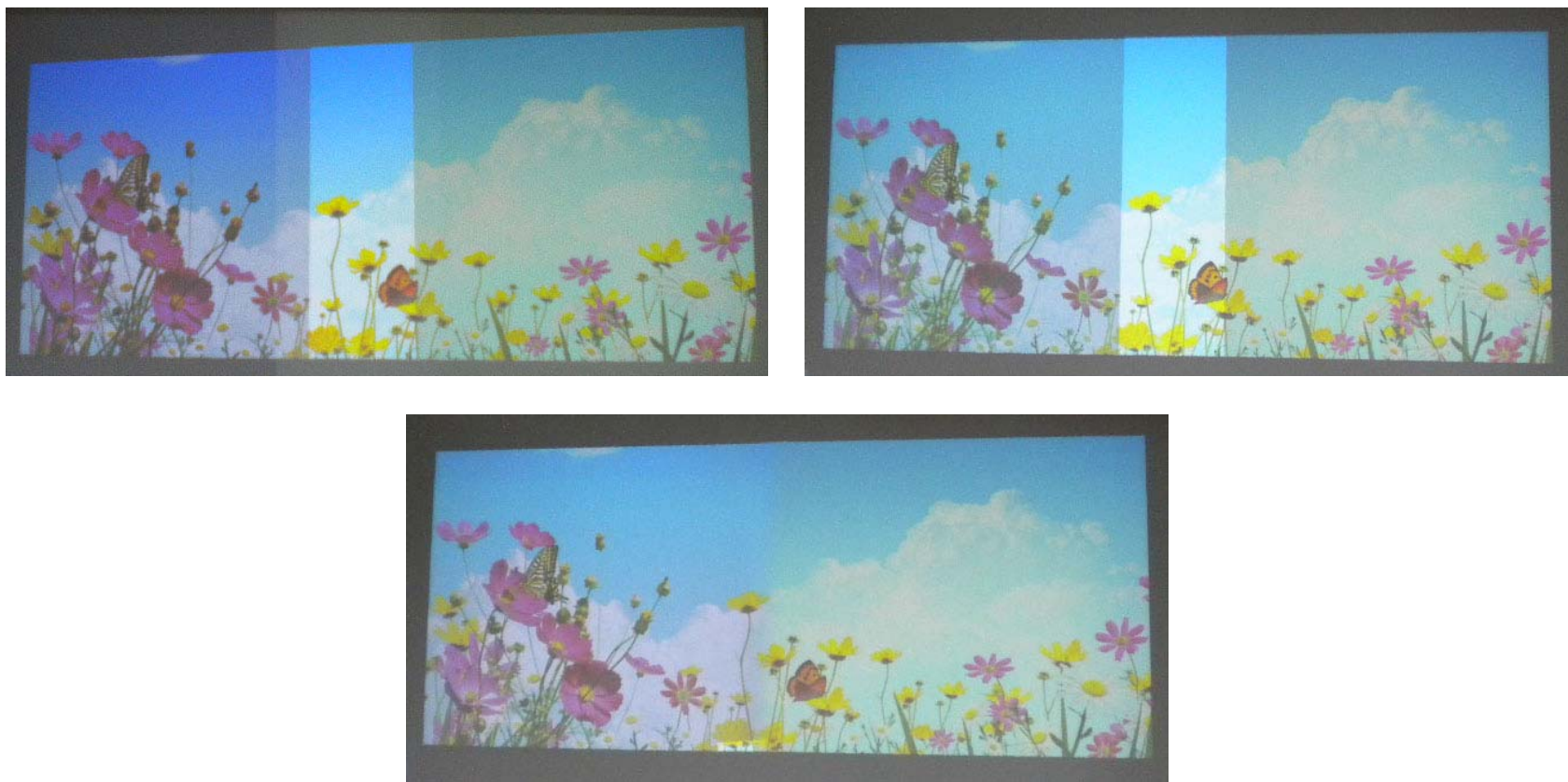

Figure 7. The left is the projected image before the color calibration and alpha blending; The right is the projected image after the color calibration but before alpha blending; The below is the projected image after the color calibration and alpha blending.

\section{Conclusions}

The color calibration as introduced and studied in this paper is very important in the large plane multi-projector displays. The paper presents an approach of color calibration using cubic B-spline. We take channel $R$ as an example and describe the steps of processing in detail, and channels $G$ and $B$ are similar to it. Because of the advantages of B-spline, it has played an important role in constructing the color transfer function of projector. After this, we also can adjust the brightness, contrast, and gamma value to make the color calibration well, and the experiment results show that it works very well. Finally, we also describe the alpha blending which can eliminate the bright band effect in the overlapping region, and do some relevant experiments. It is worth noting that the blending region is not the overlapping region in the experiment. The width of the blending region is required to be chosen appropriately to achieve seamless tiled.

\section{Acknowledgements}

This work is supported by National Natural Science Foundation (60973060), the Research Fund for the Doctoral Program (20080004001), and the Fundamental Research Funds for Central Universities (2009YJS025).

\section{REFERENCES}

[1] A. Majumder, Z. He, H. Towles and G. Welch, "Achieving Color Uniformity across Multi-Projector Displays," Proceedings of IEEE Visualization Conference, Salt Lake City, 8-13 October 2000, pp. 117-124.

[2] G. Wallace, H. Chen and K. Li, “Color Gamut Matching 
for Tiled Display Walls," Eurographics Workshop on Virtual Environments, Zurich, 22-23 May 2003, pp. 293302.

[3] M. C. Stone, "Color and Brightness Appearance Issues in Tiled Displays. Computer Graphics and Applications,” Computer Graphics and Applications, Vol. 21, No. 5, 2001, pp. 58-66. doi:10.1109/38.946632

[4] A. Majumder, D. Jones, M. McCrory, M. E. Papka and R. Stevens, "Using a Camera to Capture and Correct Spatial Photometric Variation in Multi-Projector Displays,” Proceedings of IEEE International Workshop on Projector-Camera Systems, Nice, France, 12 October 2003, pp. 1-8.

[5] M. Brown, A. Majumder and R. Yang, "Camera-Based
Calibration Techniques for Seamless Multiprojector Displays," Visualization and Computer Graphics, Vol. 10, No. 5, 2005, pp. 193-206. doi:10.1109/TVCG.2005.27

[6] J. P. Li, "Principles of Computer Graphics Tutorial," Electronic Science and Technology University Press, Chengdu, 1998.

[7] G. P. Yang and F. M. Chen, "The Gamma Correction for Multiple Projectors after Alpha Blending,” Computer Applications and Software, Vol. 26, No. 1, 2009, pp. 232233

[8] S. F. Huang, F. M. Chen and X. C. Chen, "The Application of Alpha Blending to Seamless Projection," Computer Applications and Software, Vol. 24, No. 12, 2007, pp. 161-162. 